\title{
Une recherche élémentaire pour les praticiens : les livrets de l'Ifrée
}

\section{Annie Bauer et Jean-Étienne Bidou}

\section{(2) OpenEdition}

\section{Journals}

Édition électronique

URL : http://journals.openedition.org/ere/361

DOI : $10.4000 /$ ere.361

ISSN : 2561-2271

Éditeur

Centr'ERE

Édition imprimée

Date de publication : 1 septembre 2016

ISSN : 1373-9689

Référence électronique

Annie Bauer et Jean-Étienne Bidou, « Une recherche élémentaire pour les praticiens : les livrets de I'Ifrée », Éducation relative à l'environnement [En ligne], Volume 13 - 1| 2016, mis en ligne le 15 septembre 2016, consulté le 21 février 2020. URL : http://journals.openedition.org/ere/361 ; DOI : $10.4000 /$ ere. 361 


\title{
Une recherche élémentaire pour les praticiens : les livrets de l'Ifrée
}

\author{
Annie Bauer et Jean-Étienne Bidou
}

1 L'Ifrée a toujours eu au rang de ses ambitions, celle d'apporter des ressources utiles aux praticiens de l'éducation à l'environnement. Dès les années 1990, l'association publiait des fiches thématiques ${ }^{1}$ en format court, faisant le point sur une question et destinées à outiller a minima les éducateurs qui souhaiteraient se lancer dans un projet sur la thématique, qu'elle soit environnementale ou éducationnelle.

2 Sans renoncer tout de suite à ce format et ce type de thèmes, il nous était apparu, au contact des praticiens et notamment à travers les retours de ceux qui fréquentaient les stages de l'Ifrée, qu'il existait une demande pour des études plus approfondies sur des questions émergentes. En 2008, au moment de cette réflexion, l'Ifrée et ses partenaires étaient fortement engagés dans le domaine des expositions et le problème de l'écoconception des supports pédagogiques utilisés par les praticiens ressortait particulièrement : comment faire pour avoir des outils en cohérence avec le discours? L'Ifrée saisit alors l'occasion pour lancer ce qui deviendra sa collection de livrets.

3 Sur ce sujet, il s'agissait à la fois de pouvoir caractériser les pratiques existantes, d'identifier les questions que se posaient les praticiens et de repérer les éléments mutualisables dans les pratiques qui semblaient les plus avancées. Ces trois aspects allaient devenir la colonne vertébrale du travail sur les livrets. Avec ce premier numéro s'est donc construite la méthodologie qui perdure encore aujourd'hui (à quelques variantes près) et que nous détaillons ci-après. Mais ce travail, orienté vers les praticiens, et particulièrement ceux du mouvement associatif, constitue-t-il pour autant une « recherche »?

4 Poser cette question ne vise pas à attribuer quelque degré de scientificité à des travaux dont le but fondamental est le recueil et la diffusion de pratiques, mais d'explorer à partir d'un cas spécifique le débat particulièrement actif, dans les années 1990 et 2000 et encore aujourd'hui, à propos de l'articulation entre la recherche et la pratique : la recherche pour la production de connaissances au service des pratiques éducatives ou la recherche pour la création de modèles dans des cadres théoriques précis à 
destination d'une communauté académique ? La question est d'autant plus vive que les praticiens qui disposent de capacités à se mettre en position de recherche le font quelquefois et produisent éventuellement des textes. Qu'en est-il alors de leurs productions? Existe-t-il comme le soutient Soëtard (2004), un fossé infranchissable entre chercheurs et praticiens? Ou s'agit-il au contraire d'un continuum entre recherche académique et "posture de recherche» des praticiens. C'est donc pour explorer cet entre-deux, à partir d'un cas concret, que la construction des livrets de l'Ifrée peut être analysée et qu'on peut se demander dans quelle mesure elle peut relever d'une démarche de recherche.

\section{Les livrets de l'Ifrée}

5 En 2016, on compte sept numéros, publiés au rythme d'un numéro par an depuis 2010. Il en existe une version papier, mais également une version numérique, téléchargeable sur le site de l'Ifréé2.

6 La structure de ces livrets peut varier selon les numéros et les thématiques, mais il s'agit généralement d'un petit ouvrage d'une centaine de pages dont une partie, généralement la moitié, est consacrée à la description d'expériences, dérivée des questionnaires et des entretiens menés avec les porteurs de projets ${ }^{3}$ («Ils racontent leur expérience »), suivie d'une partie synthétique à l'organisation plus variée, mais toujours orientée vers l'action («Comment s'y mettre?» devenue «Des points de repères pour agir »). Une bibliographie succincte, incluant de la littérature grise ou des sites internet, est conçue comme un outillage pour les praticiens et non comme une liste de références pour des chercheurs.

Un certain nombre de ces livrets ont donné lieu à des communications en colloques et à des publications scientifiques ${ }^{4}$. La distinction est donc assez claire entre l'esprit du livret et la publication scientifique qui relève d'une autre nature ou, du moins, d'une autre culture épistémologique.

\section{Des questions de recherche?}

8 Pour Vial (2000), la recherche se distingue de l'étude par le fait qu'elle a prétention à être reconnue comme scientifique, qu'elle est dirigée vers une communauté scientifique, même si son public peut être beaucoup plus large. La question de la reconnaissance du caractère scientifique par une communauté de pairs est essentielle : elle se fait soit par des soutenances de travaux, soit par les processus d'évaluation ou le système de références, les articles et les ouvrages étant cités ou non par la communauté scientifique.

9 Mais si cet auteur est relativement restrictif sur la nature de la recherche, il est plus inclusif dans ses critères de validité du savoir produit. Au-delà du seul critère de la méthode, il faut s'attacher à l'intérêt du thème étudié, mais aussi au sens des résultats, non seulement pour la communauté scientifique, mais aussi pour les professionnels concernés (Vial, 2000, p. 136), finalement, à la finalité de la recherche, à son dessein d'utilité sociale. C'est à ces critères qu'on peut étalonner le projet des livrets de l'Ifrée et décider s'il s'agit d'études ou de recherches. 


\section{Le choix des thèmes : entre utilité et opportunité}

$10 \mathrm{Au}$ fil des livrets s'est dessinée une ligne éditoriale des questions traitées qui permet de les définir comme suit : on cherche dans nos réseaux de partenaires un thème qui soit une question émergente pour nos publics, essentiellement animateurs d'associations et chargés de projets environnementaux dans les collectivités locales. Il peut s'agir de questions nouvelles. C'était le cas du premier livret avec la question de l'éco-conception des supports pédagogiques utilisés par les praticiens. Cela est également vrai du livret $\mathrm{n}^{\circ} 2$ sur les sciences participatives: les associations voient monter ce phénomène nouveau d'associer des citoyens à des campagnes de production de données naturalistes et se demandent ce que cela recouvre et comment l'investir utilement pour eux et leurs publics. D'autres livrets privilégient des questions plus classiques, mais pour lesquelles la réflexion et les outils mis à la disposition des praticiens manquent.

11 La thématique est précisée après les premiers échanges avec les opérateurs du champ. Pour le livret 4 sur la question des publics exclus, par exemple, nous avons balayé un grand nombre de types d'exclusion: extrême (sans abri ou milieu carcéral, par exemple, liée au handicap mental ou physique, ou liée à la précarité économique et sociale. C'est cette dernière que nous avons retenue, la première concernant un nombre trop faible d'expériences et amenant des spécificités trop fortes pour être confondues avec les autres, la deuxième ayant déjà fait l'objet de nombreuses réflexions et publications ${ }^{5}$. Encore, pour le livret 5 sur les outils numériques, l'étude de leur usage en situation d'animation a été préférée à celle de leur usage pour l'animation des réseaux, question déjà bien balisée par des acteurs reconnus dans ce domaine.

12 La mise au point du sujet, qui correspond généralement à une demande de nos publics se poursuit ainsi dans le dialogue avec ceux-ci.

\section{Repérage des expériences et des questions principales des praticiens}

13 La procédure a varié au cours du temps et en fonction des thématiques, mais s'est progressivement stabilisée.

14 Pour le premier numéro, cette opération a pris la forme d'un questionnaire envoyé tous azimuts. Cette démarche était adaptée à la question de l'éco conception. On trouvait en effet très peu d'expériences qui se déclaraient comme relevant de l'éco-conception et nous souhaitions donc interroger les pratiques de conception en général des acteurs de l'éducation à l'environnement, mais également des acteurs professionnels de la conception d'outils et d'expositions liés au monde de la culture (à travers un partenariat avec l'office de Coopération et d'Information Muséales, organisme public français, au service des musées et centres de culture scientifique et technique), qui n'abordent pas forcément le thème de l'environnement ou du développement durable, mais se sont intéressés à la question de l'éco-conception.

Dès le deuxième numéro, c'est un appel à intérêt et à expériences qui était envoyé sur les différentes listes de diffusion auxquelles nous avons accès (et particulièrement la riche et réactive liste des adhérents du réseau École et Nature). En réponse, et avec des recherches indépendantes et complémentaires, une soixantaine de programmes correspondant à nos critères (ouverts aux novices et leur proposant de produire une donnée liée aux sciences naturalistes) pouvait être identifiée. Cela constituait une base 
de données fiable pour en extraire dix-huit expériences choisies pour leur importance (tous les grands programmes nationaux doivent être présents) ou leur représentativité de la diversité des programmes repérés.

Pour les livrets suivants, c'est cette même façon de procéder qui est utilisée : appel à intérêt et à expériences et prospection pour définir les expériences à enquêter, et choix d'expériences permettant de couvrir le plus de cas de figure rencontrés (dans la limite du domaine défini). À partir des retours à cet appel nous pouvons donc à la fois identifier la pertinence du sujet abordé pour les praticiens (selon les questions qu'ils se posent et l'importance qu'ils lui accordent), affiner les problèmes qu'ils soulèvent (ce qui permet d'orienter les points à aborder dans le livret) et repérer des expériences qui pourraient être présentées dans le livret.

\section{Entre questionnaires et entretiens : des libertés avec la méthode scientifique}

La méthode est la pierre de touche de la recherche. Pour Van Der Maren (1996), elle constitue le critère fondamental de la scientificité. Il faut construire un système qui produise des données valides sur un objet de recherche, des techniques de traitements qui transforment les données en résultats et des procédures d'interprétation des résultats. Vial (op. cit.), pour qui la définition de la recherche est plus large, n'en est pas moins très strict sur les procédures de recueil et d'interprétation des données. C'est probablement dans ce domaine que le travail des livrets s'écarte le plus des méthodes scientifiques classiques. De fait, le but des livrets est d'exposer des projets, des actions ou des pratiques, de décrire leur impact en analysant les facteurs de réussite ou d'échec. Un des critères de l'échantillonnage est de mettre à disposition du lecteur la plus grande variété d'expériences.

\section{La taille et le tirage de l'échantillon}

Lors du premier numéro, nous avions envoyé le questionnaire à plus de 2000 adresses et avons eu un taux de retour assez faible : 48 questionnaires remplis et analysés. La grande quantité des non-réponses et la faible taille de l'échantillon ne permettaient certes pas un traitement statistique irréprochable, mais l'analyse multivariée permettait de mettre en évidence les caractéristiques de ces expériences et de bâtir quelques types.

Dès le deuxième numéro, c'est un appel à intérêt et à expériences qui sera la base (avec une recherche internet et dans les réseaux identifiés) de la réalisation d'un inventaire. Une analyse multivariée sur les 18 projets sélectionnés a permis de dégager les grands traits de ces projets et de bâtir une typologie à peu près représentative du champ (en confrontant les résultats de l'analyse à la vision globale des 60 projets repérés). Cette typologie a été le fondement de plusieurs interventions dans des colloques et séminaires et surtout dans des rencontres de professionnels.

20 L'utilisation d'un questionnaire écrit comme seul outil de description des expériences pour le premier livret a montré ses limites. Les informations sont un peu désincarnées et les questions ouvertes ne sont jamais investies de la même façon par les répondants. Cela a conduit dès le deuxième numéro à la volonté d'avoir un contact plus direct à 
travers une enquête téléphonique complémentaire (de $2 \mathrm{~h}$ en moyenne) visant à préciser les éléments d'analyse du projet et les pratiques mises en œuvre. Le choix, après appel à expériences diffusé dans nos réseaux, reste limité et exige des explorations complémentaires. Nous adoptons alors un échantillonnage dit «de convenance ", c'est-à-dire un choix limité de cas sur des critères déterminés : ici, le fait que les expériences soient représentatives de la diversité des situations prises en compte dans l'angle du sujet défini, en termes de sous-thème ou de modalités (par exemple, pour la prévention des déchets : des foyers témoins, des ateliers Do It Yourself, un repair café...). Le repérage des grandes tendances existant sur le sujet traité s'arrête quand les informations issues des retours des réseaux et des recherches sur internet commencent à se reboucler systématiquement. Ce type d'échantillonnage qui entre dans la catégorie des échantillonnages raisonnés ne peut prétendre à la représentativité au sens statistique du terme, et donc à la possibilité de dresser des conclusions sur l'ensemble de la population. Mais, est-ce si nécessaire lorsqu'on veut simplement décrire les pratiques (et en tirer des enseignements utiles aux praticiens)?

Le livret $\mathrm{n}^{\circ} 4$ sur la précarité s'est limité à 7 expériences, mais son aire d'investigation a été élargie à différentes thématiques qui ont fait l'objet chacune d'une recherche complémentaire pour étayer les points de repères un peu plus largement. Pour le livret suivant sur le numérique, nous sommes revenus à 10 expériences et avons aussi fait appel de façon plus importante à l'abondante littérature sur la question pour alimenter les enseignements. Pour le livret $n^{\circ} 6$ sur la plantation d'arbres dans les projets éducatifs, nous revenons au primat donné aux expériences de terrain et à leur analyse avec 18 expériences enquêtées de nouveau.

L'un des critères de choix est bien sûr l'intérêt des expériences, mais aussi leur complémentarité est importante : il s'agit de mettre en avant des expériences diverses, à la fois innovantes et présentant des éléments mutualisables, et aussi de repérer des lacunes à notre sens, les points de vigilance que ces pratiques appellent et les principales questions auxquelles certains praticiens demeurent confrontés.

\section{Les questionnaires et entretiens : la caractérisation des pratiques}

Les questionnaires permettent d'abord de décrire les projets. Cette description, au fil des numéros s'est affinée et vise maintenant à préciser les étapes de la vie des projets et les pratiques éducatives mises en œuvre. Les entretiens permettent d'aborder l'évaluation. Souvent, si l'évaluation des projets est faite en interne, les retours des participants peuvent aider à se rendre compte de l'efficacité des actions. Il est difficile pour la plupart de donner des indicateurs, mais cela est quelquefois possible. Dans le numéro sur les sciences participatives, les résultats de la recherche menée grâce aux observations du public ou sa fidélisation permettaient dans une certaine mesure d'estimer le succès des projets ; ou encore, la mobilisation des publics concernés par les actions d'éducation à l'environnement dans le numéro sur l'éducation à l'environnement dans un contexte de précarité.

24 De plus en plus, les méthodes, le déroulement des actions, les réajustements en fonction des difficultés sont questionnés. À partir du livret $n^{\circ} 3$, nous avons cherché à isoler des séquences caractéristiques, à analyser les postures. qui s'en réclament (qui correspondent à l'essentiel de la définition opérationnelle que 
nous avons retenue) et de les caractériser sans les juger. Par exemple, de nombreuses visions des sciences participatives s'opposaient au moment où nous avons écrit le livret $\mathrm{n}^{\circ} 2$ qui y est consacré. Il ne s'agissait pas pour nous de trancher en définissant la place du chercheur, ou si le rôle des participants doit ou non dépasser la simple récolte des données (qui étaient les principaux objets de polémiques) : mais plutôt d'aider à éclairer les différences (grâce à une typologie) et de mesurer ensuite les implications de ces différences pour définir les objectifs et les modalités les plus appropriées pour chacun des types de programmes. Dans le cas du livret sur l'accompagnement de démarches de développement durable des collectivités locales, nous avons là aussi abordé la question par l'analyse des pratiques enquêtées (parmi lesquelles celles de l'Ifrée, qui est présente dans ce champ). Là encore, une classification a permis de caractériser trois grandes familles de pratiques, plus ou moins adaptées selon l'objectif de l'accompagnateur et la demande ou le besoin de la collectivité accompagnée : un accompagnement "éducatif» venant se confronter à d'autres types, tels l'accompagnement "clé en main» ou «de service» qui répondent à d'autres préoccupations, impératifs ou conditions de mise en œuvre.

\section{Vers une praxéologie?}

C'est ainsi que, dans une certaine mesure, les livrets s'inscrivent dans une démarche praxéologique. Ils en partagent au moins une dimension, celle qui consiste à décrire les éléments de l'action, à les catégoriser (à l'aide de typologies), et à réfléchir sur ces éléments et leur agencement. Cependant les règles pratiques qu'on en tire ne s'appuient pas uniquement sur l'analyse de l'action, mais également sur les points de repères qu'ont les personnes de l'Ifrée participant à cette phase d'analyse. On ne peut donc pas parler de véritable «modélisation de l'action ». Le but des livrets est plutôt celui de la mutualisation de pratiques utiles, mais aussi de points de vigilance, et qui sont structurés dans des points de repère pour l'action.

\section{Bloc de la pratique, bloc de la théorie}

L'analyse des pratiques peut être structurée à l'aide de la notion d'organisation praxéologique développée par Chevallard (1999) ${ }^{6}$. Pour reprendre sa terminologie, les livrets s'intéressent particulièrement au bloc de la praxis, c'est-à-dire aux tâches et à leur technique, tandis que le bloc du logos, la théorie, y est davantage de l'ordre de l'implicite : les références au savoir constitué restent légères, peu de développements sur la culture professionnelle des praticiens. Il est cependant un aspect du bloc du logos qui est prévalent dans la confection des livrets, c'est celui de l'éthique - nous voilà donc ici sur le terrain de l'éthos. Nous ne sommes pas une association militante du secteur de l'environnement, mais nous militons tout de même pour une certaine approche éducative. Si au cours de la phase d'entretien, nous adoptons une attitude neutre, nous n'attribuons pas la même valeur à tous les propos recensés. Les contenus de la partie "points de repères pour l'action " sont donc les éléments d'analyse que nous portons sur les expériences recensées et choisies (avec toutes les précautions liées au fait que nous n'en avons qu'une connaissance partielle) ou du moins, ce qu'elles nous inspirent. Ils reflètent les points relevés par les praticiens enquêtés qui confortent notre vision ou la renouvellent dans un sens qui nous semble pertinent. 


\section{Conscientisation de l'agir?}

différent afin de réduire l'écart entre les savoir-faire et la théorie. Pour L'hôtelier et StArnaud (1994), la praxéologie est une démarche construite d'autonomisation et de conscientisation de l'agir. Dans la ligne du praticien réflexif (Schön, 1994), il s'agit de mener une réflexion consciente sur son action de façon à intervenir de la façon la plus efficace possible dans une situation particulière. La praxéologie est ainsi proche des démarches de recherche-action. Cherchant à rééquilibrer les rapports entre praticiens et chercheurs, la praxéologie s'est développée dans le domaine de l'animation socioculturelle. Pour Maurel (2000), dans le domaine de l'éducation, tout particulièrement de l'Éducation Populaire, la praxéologie s'attache à «la construction des savoirs de la pratique validés par l'expérience, modélisés, et donc transférables et utilisables par d'autres ». Il s'agit bien du but des livrets de l'Ifrée qui cherchent à reproblématiser les questions abordées, acquérir une vision synthétique du champ et réinvestir les expériences dans le champ de l'éducation à l'environnement.

Cependant, si les acteurs répondant à l'entretien exercent bien un retour réflexif sur la menée de leur projet, on ne se situe pas dans le cadre d'une démarche praxéologique. Pas de réflexion dans l'action, mais au mieux, une évaluation réflexive d'un projet passé ; pas de réflexion collective d'autonomisation et de conscientisation de l'agir en vue de modifier une situation, mais un dialogue entre la rédactrice du livret et le porteur du projet objet de l'entretien. Une démarche praxéologique du type "praticien réflexif" s'installe dans le temps long, analyse des situations éducatives complexes de façon critique, ce qui n'est pas le cas ici. Il n'est pas exclu que cette situation évolue, mais pour le moment, elle n'est pas le but de la rédaction du livret.

\section{Des liens ambigus avec la recherche académique}

L'objectif principal est donc de produire un travail lisible et utile aux praticiens, et non pas forcément irréprochable du point de vue scientifique. Malgré tout, la recherche de la qualité est une constante pour les numéros successifs du livret. Ainsi on retrouve ici une ligne de démarcation assez incertaine entre étude et recherche. Depuis le troisième numéro, le travail autour du livret bénéficie d'échanges avec une chercheure en Sciences de l'éducation. Ceux-ci se traduisent par une relecture attentive de la partie concernant les enseignements tirés des expériences et aboutit à des réactions de fond sur les contenus et à des orientations bibliographiques complémentaires. De fait, la revue bibliographique s'enrichit au fil des numéros.

31 La nécessité ressentie au bout des cinq premiers numéros, a été de renouveler nos grilles de lectures alimentant les points de repères fournis dans les livrets. Cela impliquait d'associer des personnes issues du monde de la recherche davantage en amont, au moment de l'analyse des questionnaires et même dès le cadrage de la thématique. C'est le chemin qui a été pris à partir du livret 6 . L'Ifrée devrait aujourd'hui remettre sur pied un conseil scientifique qui pourrait alimenter un groupe de travail mobilisable à différentes étapes pour les livrets à venir. Ceux-ci pourraient ainsi bénéficier d'une plus grande richesse de points de vue dans la définition du thème et d'une plus grande rigueur scientifique dans le choix des expériences à enquêter. On

Éducation relative à l'environnement, Volume 13 - 1 | 2016 
pourrait espérer aussi une plus grande pertinence dans la conception des éléments du questionnaire et de l'enquête (dont on mesure aujourd'hui les limites) et de nouvelles grilles de lecture pour leur analyse.

Il reste que si le livret prend davantage en compte l'état de la recherche et ses exigences, la préoccupation principale reste centrée sur les questionnements des praticiens et que l'objet même des livrets est de décrire et d'analyser leurs pratiques, et d'en tirer des enseignements pour ceux qui aborderaient cette question par la suite.

\section{Conclusion}

Les livrets ont été créés pour traiter des questions qui intéressent les praticiens dans leur domaine de travail, en leur proposant des ressources pour les aider à se lancer dans leur projet. En sélectionnant les pratiques qui semblent les plus caractéristiques, en les décrivant, en analysant les contextes dans lesquelles elles se trouvent, en les confrontant à des grilles de lecture, il semble possible d'en tirer des points de repères à différents niveaux, du montage du projet aux postures éducatives. Démarrés comme un travail destiné aux praticiens, ces livrets comportent des éléments de formalisation et de théorisation; ils offrent des grilles de lecture. Dès lors, ils posent la question de leur rapport à la recherche.

Mais à quelle recherche? Les livrets utilisent assez librement les outils fondamentaux de la recherche qualitative en sciences sociales: questionnaires et entretiens. Ceci invaliderait les résultats si la démarche devait souscrire au paradigme classique de la science néo-positiviste. Mais celle-ci n'est plus incontestée dans le domaine de l'éducation, et souvent même dans celui des sciences sociales. Le développement des praxéologies depuis les années 1990 semble en outre autoriser les praticiens à analyser eux-mêmes leurs propres pratiques. Cependant, selon l'expérience des livrets, la démarche praxéologique, bien que largement entamée, ne peut généralement aboutir dans les milieux d'action éducative, faute de temps, faute d'un dispositif adéquat qui permette aux praticiens un véritable travail réflexif.

Dès lors, il faut reconnaitre que la recherche autour des livrets reste limitée et sa démarche peu stable tentant de maintenir un équilibre fragile entre une exigence de rigueur, sans se couper de l'action des associations, qui nourrit ses travaux et en est le destinataire privilégié.

\section{BIBLIOGRAPHIE}

Bauer, A. et Bidou, J.E. (2011). Éco-conception et éco-concepteurs: pratiques et attitudes dans les structures éducatives et culturelles. Dans Chaumier S. et Porceda, A. (dir.) Musées et développement durable, Collection Musées-Mondes. Paris : La Documentation française.

Chevallard, Y. (1997). Familière et problématique, la figure du professeur. Recherche en Didactique des Mathématiques, 17(3), 17-54. 
Chevallard, Y. (1999). L'analyse des pratiques enseignantes en théorie anthropologique du didactique. Recherches en didactique des mathématiques, 19(2), 221-265.

Grelier, F. (2010). Vers une théorie de l'action associative : la praxis de l'éducation populaire : l'étude de cas de l'animation socioculturelle citoyenne. Thèse de Doctorat, Sciences de l'Éducation. Université Rennes 2 ; Université Européenne de Bretagne.

L'hôtellier, A. et St-Arnaud, Y. (1994). Pour une démarche praxéologique. Nouvelles pratiques sociales, 7(2), 93-109.

Maurel, C. (2000). Éducation populaire et travail de la culture. Éléments d'une théorie de la praxis. Paris : L'Harmattan.

Schön, D. A. (1994). Le praticien réflexif. Montréal : Les Éditions logiques.

Soëtard, M. (2004). Pratiques éducatives et recherche, Éduquer, 8, Consulté le 10 octobre 2016. http://rechercheseducations.revues.org/348

Van der Maren, J.-M. (1996). Méthodes de recherche pour l'éducation. (2e édition). Montréal/ Bruxelles : PUM et de Boeck.

Vial, M. (2000). La recherche en sciences de l'éducation et la santé. Spirale - Revue de Recherches en Education, 25,119-142.

\section{NOTES DE FIN}

1. Sorte de dossier thématique accompagnant la lettre d'information de l'Ifrée sur la formation en éducation à l'environnement. À l'époque de leur diffusion, le format des fiches thématiques était volontairement court (4 pages, puis 6 pages) car il nous semblait que le monde de l'éducation à l'environnement était peu enclin à la lecture d'articles longs. Le dossier présentait un point sur la question, des expériences et des outils ainsi que des ressources en termes de bibliographie, de sites internet, d'organismes de référence, d'événements ou de formations.

2. http://ifree.asso.fr

3. Des collectivités, des établissements publics, le monde de l'enseignement, avec souvent une majorité d'associations

4. Pour le $n^{\circ} 1$ : Bauer, A. et Bidou, J.E. (2011). Pour le $n^{\circ} 3$, une communication au WEEC 2014 : Bauer, A. et Bidou, J. E. (2014). Intervenir auprès de publics en précarité en France, Marrakech (Maroc).

5. Notamment par l'ATEN (Atelier Technique des Espaces Naturels) qui a pour mission de contribuer à la sauvegarde des espaces et du patrimoine naturels, par la mise en réseau et la professionnalisation des acteurs.

6. Pour Chevallard (1997, p. 38), «l'activité des personnes occupant une position donnée se décline en différents types de tâches, accomplies au moyen d'une certaine manière de faire ou technique. Ce couple constitue un savoir-faire. Mais un tel savoir-faire ne saurait vivre à l'état isolé : il appelle un environnement technologique logico-théorique ou savoir (au sens restreint), formé d'une technologie, "discours " rationnel (logos) censé justifier et rendre intelligible la technique (tekhnê), à son tour justifié et éclairé par une théorie. Le système de ces quatre composantes, constitue alors une organisation praxéologique ou praxéologie, structure bifide avec sa partie pratico-technique du savoir-faire, de l'ordre de la praxis, et sa partie technologico-th éorique, le savoir, de l'ordre du logos». 
INDEX

Keywords : practitioners, praxeology, teaching tool, mutualisation

Mots-clés : Institut de formation et de recherche en éducation à l'environnement, livrets de l'Ifrée, praticiens, praxéologie, mutualisation, outil pédagogique

\section{AUTEURS}

\section{ANNIE BAUER}

Diplômée d'études supérieures spécialisées en environnement, est chargée de mission à l'Ifrée depuis 20 ans, où elle développe la production de ressources à destination des praticiens de l'éducation à l'environnement ainsi que l'accompagnement de partenaires techniques pour la création de supports de sensibilisation et la formation dans différents domaines

\section{JEAN-ÉTIENNE BIDOU}

Géographe de formation, est chargé de la mission recherche à l'Ifrée 